WSRC-TR-94-0311

Rev 0

June 1994

\title{
Sampling and Analysis of Soil from the Old F-Area Effluent Ditch and its Surrounding Wetlands (U)
}

K. L. Dixon

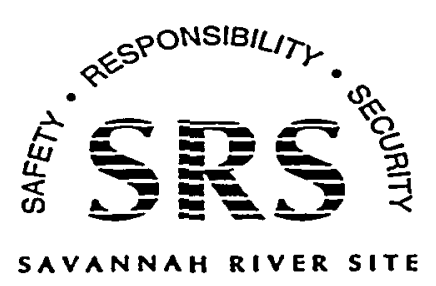

Prepared for the U.S. Department of Energy under contract no. DE-AC09-89SR18035

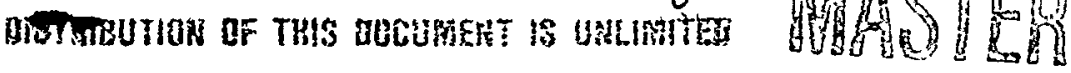




\section{DISCLAIMER}

This report was prepared as an account of work sponsored by an agency of the United States Government. Neither the United States Government nor any agency thereof, nor any of their employees, makes any warranty, express or implied, or assumes any legal liability or responsibility for the accuracy, completeness, or usefulness of any information, apparatus, product, or process disclosed, or represents that its use would not infringe privately owned rights. Reference herein to any specific commercial product, process, or service by trade name, trademark, manufacturer, or otherwise does not necessarily constitute or imply its endorsement,. recommendation, or favoring by the United States Government or any agency thereof. The views and opinions of authors expressed herein do not necessarily state or reflect those of the United States Government or any agency thereof.

This report has been reproduced directly from the best available copy.

Available to DOE and DOE contractors from the Office of Scientific and Technical Information, P.O. Box 62, Oak Ridge, TN 37831; prices available from (615) 576-8401.

Available to the public from the National Technical Information Service, U.S. Department of Commerce, 5285 Port Royal Road, Springfield, VA 22161. 


\section{DISCLAIMER}

Portions of this document may be illegible in electronic image products. Images are produced from the best available original document. 


\title{
Sampling And Analysis Of Soil From the Old F-Area Effluent Ditch and its Surrounding Wetlands (U)
}

\author{
K. L. Dixon \\ Savannah River Technology Center \\ Westinghouse Savannah River Company \\ Aiken, SC 29802
}

\section{EXECUTIVE SUMMARY}

Four surface soil samples were collected from the wetlands at the old F-Area effluent ditch. All samples were collected near shallow well point locations (Dixon et al., 1994) except FHB012, which was collected from the effluent ditch stream sediment. Samples were analyzed for metals, Target Compound List (TCL) volatile organic compounds (VOCs), and gross radiological indicators.

Barium, beryllium, and zinc were detected in all four samples and antimony was detected in three of four samples. These metals occur naturally in the wetland soils at the SRS. Comparisons of metals concentrations were made to concentration ranges taken from background wetland soil samples (Dixon et al., in prep). These comparisons showed that barium and beryllium concentrations were within expected ranges while zinc and antimony concentrations were elevated above expected concentration ranges.

Volatile organic compounds were detected in all four samples. Detected compounds included acetone, 2-butanone (Methyl ethyl ketone), chloromethane, cis-1,2-dichloroethene, and toluene. The only radionuclide detected in significant quantities was tritium which was detected in all four samples.

\section{INTRODUCTION}

The Radioactive Waste Burial Grounds are central waste storage sites that are used for the disposal of both radioactive and nonradioactive wastes originating from both onsite and offsite (Jaegge et al., 1985). The burial grounds are divided into three areas: (1) Building 643-E (old burial ground), which received wastes from 1952 through 1972; (2) Building 643-7E, which has been receiving wastes since 1969; and (3) Building 643-28E which is a closure area within 643-7E that is termed the Mixed Waste Management Facility (MWMF) (Jaegge et al., 1985). The sampling zone along the old F-Area effluent ditch receives discharge from shallow groundwater flowing under the southwest corner of 643-E.

Recent analytical results from shallow well point and near-surface water sampling in the wetlands that comprise the old F-Area effluent ditch have shown that tritium and small quantities of VOCs are outcropping in the area (Dixon 1993a and b; Dixon et al., 1994). Volatile organic compounds detected in shallow well point and near-surface water samples include 1,2-dichloroethylene, acetone, methyl ethyl ketone, toluene, tetrachloroethylene, trichloroethylene, and vinyl chloride.

In March 1994, soil samples were collected from several locations along the effluent ditch from which shallow well point and near-surface water samples are routinely collected (Figure 1). Table 1 lists the sampling locations. All samples were collected near shallow well point locations except FHB012 which was collected from the effluent ditch stream sediment. At each location, soil was scooped from the ground surface and placed directly into the sample containers. Sample containers were stored in coolers containing blue ice for transport to the processing area where they were maintained at $4^{\circ} \mathrm{C}$ until they were delivered to the lab for analysis.

The information in this report was developed during the course of work under Contract No. DE-AC09-89SR18035 with the U. S. Department of Energy. 
Analytical services were provided by the Savannah River Technology Center (SRTC) Analytical Development Section (ADS) for the analytes listed in Table 2. All samples were analyzed for the metals appearing in Table 2 using an Inductively Coupled Plasma (ICP) procedure (WSRC-L16.1, 1993a) except mercury which was quantified using a cold vapor Atomic Absorption (AA) procedure (WSRC-L16.1, 1993b). All samples were also analyzed for the volatile organic compounds appearing on the Target Compound List (TCL; Table 2) using Contract Laboratory Protocol (CLP) following a Gas Chromatography - Mass Spectrometry (GC-MS) procedure (WSRC-L16.1, 1993c). The practical quantitation limit (PQL) for all volatile organic analyses was $9 \mu \mathrm{g} / \mathrm{kg}$. All VOC samples were submitted in replicate.

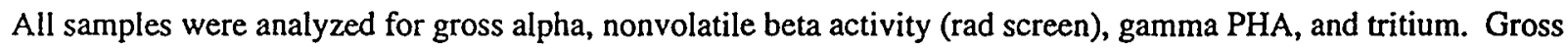
alpha and nonvolatile beta activities were determined using a microwave digestion procedure (WSRC-L16.1, 1993d). Lower limits of detection for the gross alpha and nonvolatile beta were $3 \mathrm{pCi} / \mathrm{g}$ and $7 \mathrm{pCi} / \mathrm{g}$, respectively. This method used by ADS for the rad screen is a qualitative procedure which is a good indicator of the presence of radioactivity in samples. However, due to subsampling and counting limitations, absolute quantities are subject to large uncertainties. Uncertainty estimates for gross alpha and nonvolatile beta have been estimated to be \pm 100 $\mathrm{pCi} / \mathrm{g}$. All gross alpha results are rounded up to the nearest $1 \mathrm{pCi} / \mathrm{g}$ and nonvolatile beta results are rounded up to the nearest $5 \mathrm{pCi} / \mathrm{g}$.

Samples for gamma PHA analysis were prepared using the microwave digestion procedure mentioned earlier (WSRC-L16.1, 1993d) and activities were determined using germanium detectors (WSRC-L16.1, 1993e). Minimum detectable amounts (MDA) are radionuclide specific and are identified upon determination of particular radionuclides.

Samples for tritium analysis were prepared by leaching $2.5 \mathrm{~g}$ soil samples with nitric acid. Two milliliter aliquots of the leachate solution were added to liquid scintillation cocktails and counted in a liquid scintillation counter. The MDA for tritium analyses is moisture content dependent and is estimated on a wet weight basis to be about $1 \mathrm{pCi} / \mathrm{ml}$. 


\section{Old F-Area Effluent Ditch}

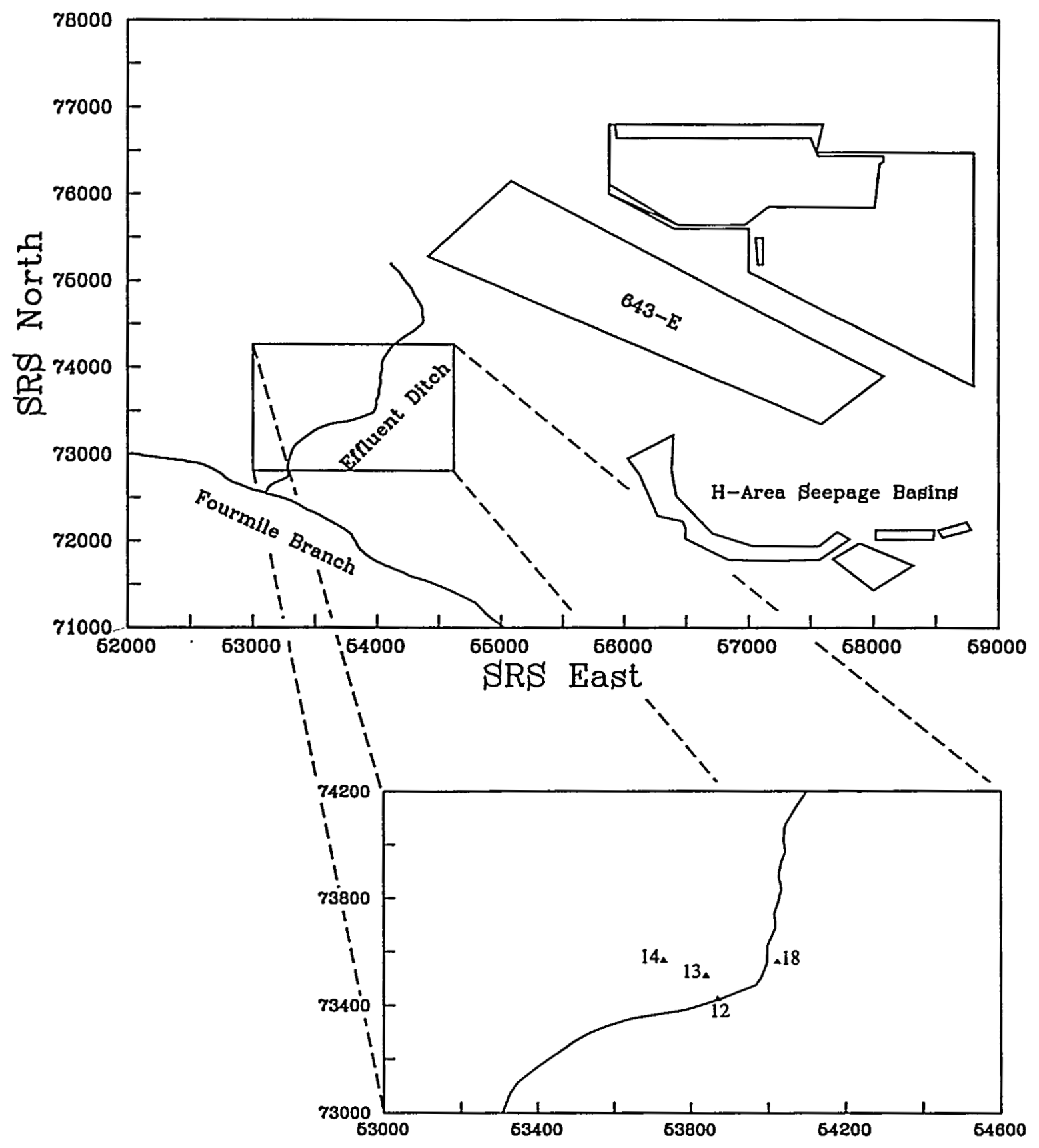

Figure 1. Soil Sampling Locations Along the Old F-Area Effluent Ditch. (All locations are prefixed by "FHB0"). 
Table 1. Soil Sampling Locations.

\begin{tabular}{lcc} 
Sampling Locations & \multicolumn{2}{c}{ SRS Coordinates } \\
\cline { 2 - 3 } & North & East \\
\hline FHB012 & 73424 & 53878 \\
FHB013 & 73514 & 53840 \\
FHB014 & 73571 & 53729 \\
FHB018 & 73567 & 54024 \\
\hline
\end{tabular}

IFHB012 is a stream sediment sample.

Table 2. List of Analytes

\begin{tabular}{|c|c|c|c|}
\hline Metals & \multicolumn{2}{|c|}{ Volatile Organic Compounds 1} & Radiological Screens \\
\hline Antimony & Chloromethane & Trichloroethene & Gross alpha \\
\hline Arsenic & Vinyl Chloride & 1,2-Dichloropropane & Nonvolatile beta \\
\hline Barium & Bromomethane & Bromodichloromethane & Gamma PHA \\
\hline Beryllium & Chloroethane & 4-Methyl-2-Pentanone & \\
\hline Cadmium & Acetone & cis-1,3-Dichloropropene & \\
\hline Chromium & 1,1-Dichloroethene & Toluene & \\
\hline Cobalt & Carbon Disulfide & trans-1,3-Dichloropropene & \\
\hline Copper & Methylene Chloride & 1,1,2-Trichloroethane & \\
\hline Lead & trans-1,2-Dichloroethene & Tetrachloroethene & \\
\hline Mercury & 1,1-Dichloroethane & 2-Hexanone & \\
\hline Nickel & Vinyl Acetate & Dibromochloromethane & \\
\hline Selenium & 2-Butanone & Chlorobenzene & \\
\hline Silver & cis-1,2-Dichloroethene & Ethylbenzene & \\
\hline Thallium & Chloroform & Xylenes (Total) & \\
\hline Tin & 1,1,1-Trichloroethane & Styrene & \\
\hline Vanadium & Carbon Tetrachloride & Bromoform & \\
\hline Zinc & Benzene & 1,1,2,2-Tetrachloroethane & \\
\hline & 1,2-Dichloroethane & & \\
\hline
\end{tabular}

TTarget Compound List 


\section{DISCUSSION}

Analytical results for the soil samples collected from the wetlands along the old F-Area effluent ditch south of 643-E (old burial ground) and its surrounding wetlands are presented in Appendix A. Radiochemistry and metals results are presented on a dry weight basis. Results of the metals analyses were provided by the laboratory on a wet weight basis and converted to dry weight based on moisture contents determined from the radiological samples. Results of the volatile organic analyses are presented on a wet weight basis. Included in the laboratory analyses tables for each location and analyte is the lab that performed the analyses, any result or analytical modifiers, and the analytical results. A legend is given at the beginning of Appendix A that provides explanations for the abbreviations and modifiers used to present the data. Data presented in Appendix A are considered scoping level data.

Samples for volatile organic analysis were submitted in replicate. The results from the replicate samples are presented in the data tables along with the reference samples and are noted with an " $R$ " in the replicate field. Replicate samples for FHB014 and FHB018 were rejected due to instrument interferences and were not reported by the lab and are not listed in the data tables.

\section{Metals}

A total of four different metals were detected in the soil samples representing 24 percent of the metals analyzed. Barium, beryllium, and zinc were detected in all four soil samples and antimony was detected in all samples except FHB014. All four of these metals occur naturally in the soils of the SRS at various concentrations. To determine if the reported concentrations were elevated above expected ranges, comparisons were made to ranges determined from background wetland soil samples (Dixon et al., in prep). Dixon et al. (in prep) collected soil cores from 50 wetland locations onsite and from 25 wetland locations offsite in an effort to establish the naturally occurring geochemical and physical properties of the wetland soils occurring on the SRS. Sampling locations were selected from areas unimpacted by SRS or other commercial operations. Soil cores were collected from five different soil groupings. Each soil group consisted of similar soil series and the five groups together represented almost all of the wetland soils occurring on the SRS. Cores were divided into depth increments and samples were analyzed for a wide range of geochemical and physical parameters. A portion of the samples underwent complete RCRA Appendix IX analysis and all of the samples were analyzed for metals and general physical properties.

Concentrations of antimony, barium, beryllium, and zinc were compared to ranges determined from Dixon et al. (in prep) for soil grouping three (Fluvaquents) for the onsite locations (surface horizon). Soil grouping three was chosen because soils in the wetlands along the old F-Area effluent ditch have been delineated by Rogers (1990) as belonging to the Seventh Approximation (USDA, 1987) great group "Fluvaquents". Barium and beryllium concentrations for the four soil samples were within the range determined from Dixon et al. (in prep) for the Fluvaquents soil grouping (grouping three). Antimony and zinc concentrations were found to be elevated above the ranges reported by Dixon et al. (in prep). Table 3 shows a comparison of the results with the ranges reported by Dixon et al. (in prep).

\section{Volatile Organic and Radiological Analyses}

A total of five different volatile organic compounds were detected above the PQL $(9 \mu \mathrm{g} / \mathrm{kg})$. Acetone was detected at all locations. 2-Butanone (methyl ethyl ketone) was detected above the PQL at locations FHBO14 and FHBO18.

Chloromethane and toluene were detected at location FHB018 and cis-1,2-dichloroethene were detected at location FHB012. Although below the PQL, toluene was observed at locations FHB012, FHB013, and FHB014 and cis-1,2dichloroethene was observed slightly below the PQL at location FHB014.

Acetone is a common laboratory contaminant and is routinely detected in method blanks. Toluene and 2-butanone are also laboratory contaminants but are not detected in method blanks as commonly as acetone. The positive acetone, 2butanone, and toluene results were reviewed with ADS and determined to be true positive values. These compounds were not detected in any of the method blanks analyzed with the samples and detected quantities were determined to be too large to be attributable to lab contamination. Each of these compounds have been observed in water samples collected from the shallow well points and near-surface water samplers installed in the wetlands along the old F-Area effluent ditch. This area is being influenced by a VOC plume migrating from 643-E which is likely the source of the organics observed in the soil samples.

Gross alpha, nonvolatile beta, and gamma PHA scans were performed on all four soil samples. Results of the gross alpha and nonvolatile beta scans were reviewed with ADS and determined to be within the range commonly observed for background soil samples at SRS. Results for gross alpha and nonvolatile beta from Dixon et al. (in prep) were not available for comparisons. Gamma PHA scans on all samples did not produce any positive results. 
Table 3. Comparison of metals concentrations with ranges taken from Dixon et al. (in prep).

\begin{tabular}{lllll} 
Location & Analyte & Result ${ }^{1}$ & Background Range $^{2}$ & Units \\
\hline FHB012 & Antimony & 44 & $<1.83-<26.3$ & $\mu \mathrm{g} / \mathrm{g}$ \\
FHB012 & Barium & 0.6 & $4.7-90.3$ & $\mu \mathrm{g} / \mathrm{g}$ \\
FHB012 & Beryllium & 0.1 & $<0.09-<0.95$ & $\mu \mathrm{g} / \mathrm{g}$ \\
FHB012 & Zinc & 73 & $1.63-9.6$ & $\mu \mathrm{g} / \mathrm{g}$ \\
FHB013 & Antimony & 70 & $<1.83-<26.3$ & $\mu \mathrm{g} / \mathrm{g}$ \\
FHB013 & Barium & 5.6 & $4.7-90.3$ & $\mu \mathrm{g} / \mathrm{g}$ \\
FHB013 & Beryllium & 0.3 & $<0.09-<0.95$ & $\mu \mathrm{g} / \mathrm{g}$ \\
FHB013 & Zinc & 212 & $1.63-9.6$ & $\mu \mathrm{g} / \mathrm{g}$ \\
FHB014 & Barium & 1.4 & $4.7-90.3$ & $\mu \mathrm{g} / \mathrm{g}$ \\
FHB014 & Beryllium & 0.84 & $<0.09-<0.95$ & $\mu \mathrm{g} / \mathrm{g}$ \\
FHB014 & Zinc & 191 & $1.63-9.6$ & $\mu \mathrm{g} / \mathrm{g}$ \\
FHB018 & Antimony & 77 & $<1.83-<26.3$ & $\mu \mathrm{g} / \mathrm{g}$ \\
FHB018 & Barium & 32 & $4.7-90.3$ & $\mu \mathrm{g} / \mathrm{g}$ \\
FHB018 & Beryllium & 0.37 & $<0.09-<0.95$ & $\mu \mathrm{g} / \mathrm{g}$ \\
FHB018 & Zinc & 160 & $1.63-9.6$ & $\mu \mathrm{g} / \mathrm{g}$ \\
\hline
\end{tabular}

I Reported as dry weight.

2 Ranges presented were determined from Dixon et al. (in prep). 


\section{REFERENCES}

Dixon, K.L. 1993a. Preliminary Results of Volatile Organic Analysis of UTRC-MWMF wetland water samples. SRT-ESS-93-0658. Westinghouse Savannah River Company, Savannah River Technology Center, Aiken SC.

Dixon, K.L. 1993b. Preliminary Results Of Volatile Organic Analysis of UTRC-MWMF Wetland Water Samples. SRT-ESS-93-0751. Westinghouse Savannah River Company, Savannah River Technology Center, Aiken SC.

Dixon, K.L, C.L. Cummins, and V.A. Rogers. 1994. Quarterly Sampling of the Wetlands Along the Old F-Area Effluent Ditch: March 1994. WSRC-TR-94-0225. Westinghouse Savannah River Company, Savannah River Technology Center, Aiken SC.

Dixon, K.L., C.L. Cummins, V.A. Rogers, R.C. Tuckfield, and J.H. Weber. Geochemical and Physical Properties of Wetland Soils at the Savannah River Site. In preparation. Westinghouse Savannah River Company, Savannah River Technology Center, Aiken SC.

Jaegge, W.J., N.L. Kolb, B.B. Looney, I.W. Marine, O.A. Towler, and J.R. Cook. 1985. Radioactive Waste Burial Grounds. DPST-85-694. Savannah River Laboratory, Aiken, SC.

Rogers, V.A. 1990. Soil Survey of the Savannah River Plant Area, Parts of Aiken, Barnwell, and Allendale Counties, South Carolina. Soil Conservation Service, U.S. Department of Agriculture.

U. S. Department of Agriculture. 1987. Keys to Soil Taxonomy, Third printing. Cornell University. Ithaca, NY.

WSRC-L16.1 (Vol. 2). 1993a. ADS1500. Inductively Coupled Plasma - Atomic Emission Spectrometer ARL3580. Analytical Development Section - Analytical Operating Procedures. Westinghouse Savannah River Company, Aiken SC.

WSRC-L16.1 (Vol. 2). 1993b. ADS1505. Operating Procedure for Mercury Determination by Cold Vapor Atomic Absorption. Analytical Development Section - Analytical Operating Procedures. Westinghouse Savannah River Company, Aiken SC.

WSRC-L16.1 (Vol. 4). 1993c. EPA RCRA Organics. ADS2656. GC/MS for Volatile Organics - Contract Laboratory Protocol Methods. Analytical Development Section - Analytical Operating Procedures. Westinghouse Savannah River Company, Aiken SC.

WSRC-L16.1 (Vol. 2). 1993d. ADS2424. Gross Alpha/Beta Determination By Liquid Scintillation Counting. Analytical Development Section - Analytical Operating Procedures. Westinghouse Savannah River Company, Aiken $\mathrm{SC}$.

WSRC-L16.1 (Vol. 2). 1993e. ADS2420. Gamma Sample Preparation and Analysis (Gamma PHA). Analytical Development Section - Analytical Operating Procedures. Westinghouse Savannah River Company, Aiken SC. 
WSRC-TR-94-0311 


\section{APPENDIX A}

Analytical Results 
WSRC-TR-94-03II 


\section{ANALYTICAL RESULTS}

Analytical results for each soil sampling location are presented in this section. Included in the laboratory analyses tables for each location and analyte is the lab that performed the analyses, any result or analytical qualifiers, the analytical results and the units of measurement.

The following is a list of abbreviations used in the tables:

Lab Laboratory performing analysis

Rep Replication or Lab Duplication Sample (denoted by either a "R" or a "D")

Qualifier Result or analysis qualifier

ADS Analytical Development Section

Result and Analysis Qualifiers

\begin{tabular}{|l|l|}
\hline \multicolumn{2}{|l|}{ Result Qualifiers } \\
\hline CODE & DESCRIPTION \\
\hline (BLANK) & Data not remarked. Number should be interpreted exactly as reported. \\
\hline J & The analytical result is an estimated quantity. \\
\hline R & $\begin{array}{l}\text { Rejected because of performance requirements in the sample or associated quality contro } \\
\text { analyses were not met. The analyte may or may not be present. }\end{array}$ \\
\hline U & Material analyzed for but not detected. Value reported is the sample quantification limit. \\
\hline
\end{tabular}

Analysis Qualifiers

\begin{tabular}{l|l}
\hline CODE & DESCRIPTION \\
\hline
\end{tabular}

L Analytical result is greater than the upper calibration value (20X PQL). For most VOC analytes, linearity has been observed up to $100 \mathrm{X}$ PQL. 
FHBO12

Sample Date: 03/07/94

Matrix: Soil

Moisture Content: $30.2 \%$

Metals Analyses:

\begin{tabular}{llccc} 
Analyte & Lab & Qualifier & Results & Units 1 \\
\hline Antimony & ADS & & 44 & $\mu \mathrm{g} / \mathrm{g}$ \\
Arsenic & ADS & $\mathrm{U}$ & 29 & $\mu \mathrm{g} / \mathrm{g}$ \\
Barium & ADS & & 0.6 & $\mu \mathrm{g} / \mathrm{g}$ \\
Beryilium & ADS & & 0.1 & $\mu \mathrm{g} / \mathrm{g}$ \\
Cadmium & ADS & $\mathrm{U}$ & 1 & $\mu \mathrm{g} / \mathrm{g}$ \\
Chromium & ADS & $\mathrm{U}$ & 6 & $\mu \mathrm{g} / \mathrm{g}$ \\
Cobalt & ADS & $\mathrm{U}$ & 6 & $\mu \mathrm{g} / \mathrm{g}$ \\
Copper & ADS & $\mathrm{U}$ & 1 & $\mu \mathrm{g} / \mathrm{g}$ \\
Lead & ADS & $\mathrm{U}$ & 29 & $\mu \mathrm{g} / \mathrm{g}$ \\
Mercury & ADS & $\mathrm{U}$ & 1 & $\mu \mathrm{g} / \mathrm{g}$ \\
Nickel & ADS & $\mathrm{U}$ & 7 & $\mu \mathrm{g} / \mathrm{g}$ \\
Selenium & ADS & $\mathrm{U}$ & 43 & $\mu \mathrm{g} / \mathrm{g}$ \\
Silver & ADS & $\mathrm{U}$ & 3 & $\mu \mathrm{g} / \mathrm{g}$ \\
Thallium & ADS & $\mathrm{U}$ & 29 & $\mu \mathrm{g} / \mathrm{g}$ \\
Tin & ADS & $\mathrm{U}$ & 10 & $\mu \mathrm{g} / \mathrm{g}$ \\
Vanadium & ADS & $\mathrm{U}$ & 1 & $\mu \mathrm{g} / \mathrm{g}$ \\
Zinc & ADS & & 73 & $\mu \mathrm{g} / \mathrm{g}$ \\
\hline
\end{tabular}

$\mathrm{T}_{\text {Reported as dry weight after moisture content corrections. }}$

Volatile Organic Analyses:

\begin{tabular}{llcccc} 
Analyte & Lab & Rep & Qualifier & Results & Units 1 \\
\hline 1,1,1-Trichloroethane & ADS & & $\mathrm{U}$ & 9 & $\mu \mathrm{g} / \mathrm{kg}$ \\
1,1,1-Trichloroethane & ADS & $\mathrm{R}$ & $\mathrm{U}$ & 9 & $\mu \mathrm{g} / \mathrm{kg}$ \\
1,1,1-Trichloroethane & ADS & $\mathrm{D}$ & $\mathrm{U}$ & 9 & $\mu \mathrm{g} / \mathrm{kg}$ \\
1,1,2,2-Tetrachloroethane & ADS & & $\mathrm{U}$ & 9 & $\mu \mathrm{g} / \mathrm{kg}$ \\
1,1,2,2-Tetrachloroethane & ADS & $\mathrm{R}$ & $\mathrm{U}$ & 9 & $\mu \mathrm{g} / \mathrm{kg}$ \\
1,1,2,2-Tetrachloroethane & ADS & $\mathrm{D}$ & $\mathrm{U}$ & 9 & $\mu \mathrm{g} / \mathrm{kg}$ \\
1,1,2-Trichloroethane & ADS & & $\mathrm{U}$ & 9 & $\mu \mathrm{g} / \mathrm{kg}$ \\
1,1,2-Trichloroethane & ADS & $\mathrm{R}$ & $\mathrm{U}$ & 9 & $\mu \mathrm{g} / \mathrm{kg}$ \\
1,1,2-Trichloroethane & ADS & $\mathrm{D}$ & $\mathrm{U}$ & 9 & $\mu \mathrm{g} / \mathrm{kg}$ \\
1,1-Dichloroethane & ADS & & $\mathrm{U}$ & 9 & $\mu \mathrm{g} / \mathrm{kg}$ \\
1,1-Dichloroethane & ADS & $\mathrm{R}$ & $\mathrm{U}$ & 9 & $\mu \mathrm{g} / \mathrm{kg}$ \\
1,1-Dichloroethane & ADS & $\mathrm{D}$ & $\mathrm{U}$ & 9 & $\mu \mathrm{g} / \mathrm{kg}$ \\
1,1-Dichloroethene & ADS & & $\mathrm{U}$ & 9 & $\mu \mathrm{g} / \mathrm{kg}$ \\
1,1-Dichloroethene & ADS & $\mathrm{R}$ & $\mathrm{U}$ & 9 & $\mu \mathrm{g} / \mathrm{kg}$ \\
1,1-Dichloroethene & ADS & $\mathrm{D}$ & $\mathrm{U}$ & 9 & $\mu \mathrm{g} / \mathrm{kg}$ \\
1,2-Dichloroethane & ADS & & $\mathrm{U}$ & 9 & $\mu \mathrm{g} / \mathrm{kg}$ \\
1,2-Dichloroethane & ADS & $\mathrm{R}$ & $\mathrm{U}$ & 9 & $\mu \mathrm{g} / \mathrm{kg}$ \\
1,2-Dichloroethane & ADS & $\mathrm{D}$ & $\mathrm{U}$ & 9 & $\mu \mathrm{g} / \mathrm{kg}$
\end{tabular}


FHB012

Volatile Organic Analyses Continued:

\begin{tabular}{|c|c|c|c|c|c|}
\hline Analyte & $\mathrm{Lab}$ & Rep & Qualifier & Results & Units 1 \\
\hline 1,2-Dichloropropane & ADS & & $\mathrm{U}$ & 9 & $\mu \mathrm{g} / \mathrm{kg}$ \\
\hline 1,2-Dichloropropane & ADS & $\mathrm{R}$ & $\mathrm{U}$ & 9 & $\mu \mathrm{g} / \mathrm{kg}$ \\
\hline 1,2-Dichloropropane & ADS & $\mathrm{D}$ & $\mathrm{J}$ & 0 & $\mu \mathrm{g} / \mathrm{kg}$ \\
\hline 2-Butanone & ADS & & $U$ & 9 & $\mu \mathrm{g} / \mathrm{kg}$ \\
\hline 2-Butanone & ADS & $\mathrm{R}$ & $U$ & 9 & $\mu \mathrm{g} / \mathrm{kg}$ \\
\hline 2-Butanone & ADS & $\mathrm{D}$ & $\mathrm{J}$ & 1 & $\mu \mathrm{g} / \mathrm{kg}$ \\
\hline 2-Hexanone & ADS & & U & 9 & $\mu \mathrm{g} / \mathrm{kg}$ \\
\hline 2-Hexanone & ADS & $\mathrm{R}$ & $\mathrm{U}$ & 9 & $\mu \mathrm{g} / \mathrm{kg}$ \\
\hline 2-Hexanone & ADS & $\mathrm{D}$ & $\mathrm{U}$ & 9 & $\mu \mathrm{g} / \mathrm{kg}$ \\
\hline 4-Methyl-2-Pentanone & ADS & & $\mathrm{J}$ & 1 & $\mu \mathrm{g} / \mathrm{kg}$ \\
\hline 4-Methyl-2-Pentanone & ADS & $\mathrm{R}$ & $\mathrm{J}$ & 1 & $\mu \mathrm{g} / \mathrm{kg}$ \\
\hline 4-Methyl-2-Pentanone & ADS & $\mathrm{D}$ & U & 9 & $\mu \mathrm{g} / \mathrm{kg}$ \\
\hline Acetone & ADS & & U & 9 & $\mu \mathrm{g} / \mathrm{kg}$ \\
\hline Acetone & $\mathrm{ADS}$ & $\mathrm{R}$ & $\mathrm{J}$ & 9 & $\mu \mathrm{g} / \mathrm{kg}$ \\
\hline Acetone & ADS & $\mathrm{D}$ & & 14 & $\mu \mathrm{g} / \mathrm{kg}$ \\
\hline Benzene & ADS & & $\mathrm{J}$ & 0 & $\mu \mathrm{g} / \mathrm{kg}$ \\
\hline Benzene & ADS & $\mathrm{R}$ & $\mathrm{J}$ & 0 & $\mu \mathrm{g} / \mathrm{kg}$ \\
\hline Benzene & ADS & $D$ & $\mathrm{~J}$ & 0 & $\mu \mathrm{g} / \mathrm{kg}$ \\
\hline Bromodichloromethane & ADS & & $\mathbf{U}$ & 9 & $\mu \mathrm{g} / \mathrm{kg}$ \\
\hline Bromodichloromethane & $\mathrm{ADS}$ & $\mathrm{R}$ & $\mathrm{U}$ & 9 & $\mu \mathrm{g} / \mathrm{kg}$ \\
\hline Bromodichloromethane & $\mathrm{ADS}$ & $\mathrm{D}$ & $\mathrm{U}$ & 9 & $\mu \mathrm{g} / \mathrm{kg}$ \\
\hline Bromoform & ADS & & $\mathbf{U}$ & 9 & $\mu \mathrm{g} / \mathrm{kg}$ \\
\hline Bromoform & $\mathrm{ADS}$ & $\mathrm{R}$ & $\mathrm{U}$ & 9 & $\mu \mathrm{g} / \mathrm{kg}$ \\
\hline Bromoform & ADS & $\mathrm{D}$ & $\mathrm{U}$ & 9 & $\mu \mathrm{g} / \mathrm{kg}$ \\
\hline Bromomethane & ADS & & $U$ & 9 & $\mu \mathrm{g} / \mathrm{kg}$ \\
\hline Bromomethane & ADS & $\mathbf{R}$ & $U$ & 9 & $\mu \mathrm{g} / \mathrm{kg}$ \\
\hline Bromomethane & ADS & $\mathrm{D}$ & $\mathrm{U}$ & 9 & $\mu \mathrm{g} / \mathrm{kg}$ \\
\hline Carbon Disulfide & ADS & & $\mathbf{J}$ & 1 & $\mu \mathrm{g} / \mathrm{kg}$ \\
\hline Carbon Disulfide & ADS & $\mathrm{R}$ & $\mathbf{J}$ & 1 & $\mu \mathrm{g} / \mathrm{kg}$ \\
\hline Carbon Disulfide & $\mathrm{ADS}$ & $\mathrm{D}$ & $\mathbf{J}$ & 1 & $\mu \mathrm{g} / \mathrm{kg}$ \\
\hline Carbon Tetrachloride & $\mathrm{ADS}$ & & $U$ & 9 & $\mu \mathrm{g} / \mathrm{kg}$ \\
\hline Carbon Tetrachloride & ADS & $\mathrm{R}$ & $\mathrm{U}$ & 9 & $\mu \mathrm{g} / \mathrm{kg}$ \\
\hline Carbon Tetrachloride & ADS & $\mathrm{D}$ & $U$ & 9 & $\mu \mathrm{g} / \mathrm{kg}$ \\
\hline Chlorobenzene & ADS & & $\mathrm{U}$ & 9 & $\mu \mathrm{g} / \mathrm{kg}$ \\
\hline Chlorobenzene & ADS & $\mathrm{R}$ & $\mathrm{U}$ & 9 & $\mu \mathrm{g} / \mathrm{kg}$ \\
\hline Chlorobenzene & ADS & $\mathrm{D}$ & $U$ & 9 & $\mu \mathrm{g} / \mathrm{kg}$ \\
\hline Chloroethane & ADS & & $U$ & 9 & $\mu \mathrm{g} / \mathrm{kg}$ \\
\hline Chloroethane & ADS & $\mathbf{R}$ & $\mathrm{J}$ & 3 & $\mu \mathrm{g} / \mathrm{kg}$ \\
\hline Chloroethane & $\mathrm{ADS}$ & D & $\mathrm{U}$ & 9 & $\mu \mathrm{g} / \mathrm{kg}$ \\
\hline Chloroform & ADS & & $J$ & 0 & $\mu \mathrm{g} / \mathrm{kg}$ \\
\hline Chloroform & $\mathrm{ADS}$ & $\mathrm{R}$ & $\mathbf{J}$ & 0 & $\mu \mathrm{g} / \mathrm{kg}$ \\
\hline Chloroform & ADS & $\mathrm{D}$ & $\mathrm{U}$ & 9 & $\mu \mathrm{g} / \mathrm{kg}$ \\
\hline Chloromethane & ADS & & $\mathrm{U}$ & 9 & $\mu \mathrm{g} / \mathrm{kg}$ \\
\hline Chloromethane & ADS & $\mathrm{R}$ & $U$ & 9 & $\mu \mathrm{g} / \mathrm{kg}$ \\
\hline Chloromethane & ADS & D & $\mathrm{U}$ & 9 & $\mu \mathrm{g} / \mathrm{kg}$ \\
\hline
\end{tabular}


FHB012

Volatile Organic Analyses Continued:

\begin{tabular}{|c|c|c|c|c|c|}
\hline Analyte & Lab & Rep & Qualifier & Results & Units 1 \\
\hline cis-1,2-Dichloroethene & $\mathrm{ADS}$ & & & 9 & $\mu \mathrm{g} / \mathrm{kg}$ \\
\hline cis-1,2-Dichloroethene & ADS & $\mathrm{R}$ & $J$ & 8 & $\mu \mathrm{g} / \mathrm{kg}$ \\
\hline cis-1,2-Dichloroethene & ADS & D & & 20 & $\mu \mathrm{g} / \mathrm{kg}$ \\
\hline cis-1,3-Dichloropropene & ADS & & $\mathrm{U}$ & 9 & $\mu g / \mathrm{kg}$ \\
\hline cis-1,3-Dichloropropene & $\operatorname{ADS}$ & $\mathrm{R}$ & $\mathrm{U}$ & 9 & $\mu \mathrm{g} / \mathrm{kg}$ \\
\hline cis-1,3-Dichloropropene & ADS & $\mathrm{D}$ & $\mathrm{U}$ & 9 & $\mu \mathrm{g} / \mathrm{kg}$ \\
\hline Dibromochloromethane & ADS & & $\mathrm{U}$ & 9 & $\mu \mathrm{g} / \mathrm{kg}$ \\
\hline Dibromochloromethane & ADS & $\mathrm{R}$ & $\mathrm{U}$ & 9 & $\mu \mathrm{g} / \mathrm{kg}$ \\
\hline Dibromochloromethane & $\mathrm{ADS}$ & $\mathrm{D}$ & $\mathrm{U}$ & 9 & $\mu \mathrm{g} / \mathrm{kg}$ \\
\hline Ethylbenzene & ADS & & $\mathrm{U}$ & 9 & $\mu \mathrm{g} / \mathrm{kg}$ \\
\hline Ethylbenzene & ADS & $\mathrm{R}$ & $\mathrm{U}$ & 9 & $\mu \mathrm{g} / \mathrm{kg}$ \\
\hline Ethylbenzene & ADS & D & $\mathrm{U}$ & 9 & $\mu \mathrm{g} / \mathrm{kg}$ \\
\hline Methylene Chloride & ADS & & $\mathrm{J}$ & 0 & $\mu \mathrm{g} / \mathrm{kg}$ \\
\hline Methylene Chloride & ADS & $\mathrm{R}$ & $\mathrm{J}$ & 0 & $\mu \mathrm{g} / \mathrm{kg}$ \\
\hline Methylene Chloride & ADS & $\mathrm{D}$ & $\mathrm{J}$ & 0 & $\mu \mathrm{g} / \mathrm{kg}$ \\
\hline Styrene & ADS & & $\mathrm{U}$ & 9 & $\mu \mathrm{g} / \mathrm{kg}$ \\
\hline Styrene & $\operatorname{ADS}$ & $\mathrm{R}$ & $\mathrm{U}$ & 9 & $\mu \mathrm{g} / \mathrm{kg}$ \\
\hline Styrene & ADS & $\mathrm{D}$ & $\mathrm{U}$ & 9 & $\mu \mathrm{g} / \mathrm{kg}$ \\
\hline Tetrachloroethene & ADS & & $\mathrm{J}$ & 3 & $\mu \mathrm{g} / \mathrm{kg}$ \\
\hline Tetrachloroethene & ADS & $\mathrm{R}$ & $\mathbf{J}$ & 5 & $\mu \mathrm{g} / \mathrm{kg}$ \\
\hline Tetrachloroethene & ADS & $\mathrm{D}$ & $\mathbf{J}$ & 4 & $\mu g / \mathrm{kg}$ \\
\hline Toluene & ADS & & $\mathrm{J}$ & 1 & $\mu \mathrm{g} / \mathrm{kg}$ \\
\hline Toluene & ADS & $\mathrm{R}$ & $\mathrm{J}$ & 2 & $\mu \mathrm{g} / \mathrm{kg}$ \\
\hline Toluene & ADS & $\mathrm{D}$ & $\mathrm{J}$ & 1 & $\mu \mathrm{g} / \mathrm{kg}$ \\
\hline trans-1,2-Dichloroethene & ADS & & $\mathrm{J}$ & 0 & $\mu g / \mathrm{kg}$ \\
\hline trans-1,2-Dichloroethene & ADS & $\mathrm{R}$ & J & 0 & $\mu \mathrm{g} / \mathrm{kg}$ \\
\hline trans-1,2-Dichloroethene & ADS & $\mathrm{D}$ & $\mathrm{J}$ & 1 & $\mu \mathrm{g} / \mathrm{kg}$ \\
\hline trans-1,3-Dichloropropene & ADS & & $\mathrm{U}$ & 9 & $\mu \mathrm{g} / \mathrm{kg}$ \\
\hline trans-1,3-Dichloropropene & ADS & $\mathrm{R}$ & $\mathrm{U}$ & 9 & $\mu \mathrm{g} / \mathrm{kg}$ \\
\hline trans-1,3-Dichloropropene & ADS & $\mathrm{D}$ & $\mathrm{U}$ & 9 & $\mu g / \mathrm{kg}$ \\
\hline Trichloroethene & ADS & & $\mathrm{J}$ & 3 & $\mu \mathrm{g} / \mathrm{kg}$ \\
\hline Trichloroethene & ADS & $\mathrm{R}$ & $\mathrm{J}$ & 4 & $\mu \mathrm{g} / \mathrm{kg}$ \\
\hline Trichloroethene & ADS & $\mathrm{D}$ & $J$ & 5 & $\mu g / \mathrm{kg}$ \\
\hline Vinyl Acetate & ADS & & $\mathrm{U}$ & 9 & $\mu \mathrm{g} / \mathrm{kg}$ \\
\hline Vinyl Acetate & ADS & $\mathrm{R}$ & $\mathrm{U}$ & 9 & $\mu \mathrm{g} / \mathrm{kg}$ \\
\hline Vinyl Acetate & ADS & $\mathrm{D}$ & $\mathrm{U}$ & 9 & $\mu \mathrm{g} / \mathrm{kg}$ \\
\hline Vinyl Chloride & $\mathrm{ADS}$ & & $\mathrm{U}$ & 9 & $\mu \mathrm{g} / \mathrm{kg}$ \\
\hline Vinyl Chloride & ADS & $\mathrm{R}$ & $\mathrm{U}$ & 9 & $\mu \mathrm{g} / \mathrm{kg}$ \\
\hline Vinyl Chloride & ADS & $\mathrm{D}$ & $\mathrm{U}$ & 9 & $\mu \mathrm{g} / \mathrm{kg}$ \\
\hline Xylenes (Total) & ADS & & U & 9 & $\mu \mathrm{g} / \mathrm{kg}$ \\
\hline Xylenes (Total) & $\mathrm{ADS}$ & $\mathrm{R}$ & $\mathrm{U}$ & 9 & $\mu \mathrm{g} / \mathrm{kg}$ \\
\hline Xylenes (Total) & ADS & $\mathrm{D}$ & $\mathrm{U}$ & 9 & $\mu \mathrm{g} / \mathrm{kg}$ \\
\hline
\end{tabular}

TReported as wet weight. 


\section{FHB012}

Radiological Analyses:

\begin{tabular}{lllll} 
Analyte & Lab & Qualifier & Result & Units \\
\hline Gross Alpha & ADS & $\mathrm{J}$ & 1 & $\mathrm{pCi} / \mathrm{g}$ \\
Nonvolatile Beta & ADS & & 30 & $\mathrm{pCi} / \mathrm{g}^{1}$ \\
Tritium & ADS & & 18000 & $\mathrm{pCi} / \mathrm{g} 2$ \\
\hline
\end{tabular}

$\mathrm{I}_{\text {Reported as dry weight after moisture content corrections. }}$

2 Reported as wet weight. 
FHB013

Sample Date: 03/07/94

Matrix: Soil

Moisture Content: $21.8 \%$

Metals Analyses:

\begin{tabular}{lcccc} 
Analyte & Lab & Qualifier & Results & Units 1 \\
\hline Antimony & ADS & & 70 & $\mu \mathrm{g} / \mathrm{g}$ \\
Arsenic & ADS & $\mathrm{U}$ & 30 & $\mu \mathrm{g} / \mathrm{g}$ \\
Barium & ADS & & 5.6 & $\mu \mathrm{g} / \mathrm{g}$ \\
Beryllium & ADS & & 0.3 & $\mu \mathrm{g} / \mathrm{g}$ \\
Cadmium & ADS & $\mathrm{U}$ & 2 & $\mu \mathrm{g} / \mathrm{g}$ \\
Chromium & ADS & $\mathrm{U}$ & 6 & $\mu \mathrm{g} / \mathrm{g}$ \\
Cobalt & ADS & $\mathrm{U}$ & 6 & $\mu \mathrm{g} / \mathrm{g}$ \\
Copper & ADS & $\mathrm{U}$ & 2 & $\mu \mathrm{g} / \mathrm{g}$ \\
Lead & ADS & $\mathrm{U}$ & 30 & $\mu \mathrm{g} / \mathrm{g}$ \\
Mercury & ADS & $\mathrm{U}$ & 2 & $\mu \mathrm{g} / \mathrm{g}$ \\
Nickel & ADS & $\mathrm{U}$ & 8 & $\mu \mathrm{g} / \mathrm{g}$ \\
Selenium & ADS & $\mathrm{U}$ & 46 & $\mu \mathrm{g} / \mathrm{g}$ \\
Silver & ADS & $\mathrm{U}$ & 3 & $\mu \mathrm{g} / \mathrm{g}$ \\
Thallium & ADS & $\mathrm{U}$ & 30 & $\mu \mathrm{g} / \mathrm{g}$ \\
Tin & ADS & $\mathrm{U}$ & 11 & $\mu \mathrm{g} / \mathrm{g}$ \\
Vanadium & ADS & $\mathrm{U}$ & 2 & $\mu \mathrm{g} / \mathrm{g}$ \\
Zinc & ADS & & 212 & $\mu \mathrm{g} / \mathrm{g}$ \\
\hline
\end{tabular}

$\mathrm{T}_{\text {Reported as dry weight after moisture content corrections. }}$

Volatile Organic Analyses:

\begin{tabular}{llcccc} 
Analyte & Lab & Rep & Qualifier & Results & Units 1 \\
\hline 1,1,1-Trichloroethane & ADS & & $\mathrm{U}$ & 9 & $\mu \mathrm{g} / \mathrm{kg}$ \\
1,1,1-Trichloroethane & ADS & $\mathrm{R}$ & $\mathrm{U}$ & 9 & $\mu \mathrm{g} / \mathrm{kg}$ \\
1,1,2,2-Tetrachloroethane & ADS & & $\mathrm{U}$ & 9 & $\mu \mathrm{g} / \mathrm{kg}$ \\
1,1,2,2-Tetrachloroethane & ADS & $\mathrm{R}$ & $\mathrm{U}$ & 9 & $\mu \mathrm{g} / \mathrm{kg}$ \\
1,1,2-Trichloroethane & ADS & & $\mathrm{U}$ & 9 & $\mu \mathrm{g} / \mathrm{kg}$ \\
1,1,2-Trichloroethane & ADS & $\mathrm{R}$ & $\mathrm{U}$ & 9 & $\mu \mathrm{g} / \mathrm{kg}$ \\
1,1-Dichloroethane & ADS & & $\mathrm{J}$ & 0 & $\mu \mathrm{g} / \mathrm{kg}$ \\
1,1-Dichloroethane & ADS & $\mathrm{R}$ & $\mathrm{U}$ & 9 & $\mu \mathrm{g} / \mathrm{kg}$ \\
1,1-Dichloroethene & ADS & & $\mathrm{U}$ & 9 & $\mu \mathrm{g} / \mathrm{kg}$ \\
1,1-Dichloroethene & ADS & $\mathrm{R}$ & $\mathrm{U}$ & 9 & $\mu \mathrm{g} / \mathrm{kg}$ \\
1,2-Dichloroethane & ADS & & $\mathrm{U}$ & 9 & $\mu \mathrm{g} / \mathrm{kg}$ \\
1,2-Dichloroethane & ADS & $\mathrm{R}$ & $\mathrm{U}$ & 9 & $\mu \mathrm{g} / \mathrm{kg}$ \\
1,2-Dichloropropane & ADS & & $\mathrm{U}$ & 9 & $\mu \mathrm{g} / \mathrm{kg}$ \\
1,2-Dichloropropane & ADS & $\mathrm{R}$ & $\mathrm{U}$ & 9 & $\mu \mathrm{g} / \mathrm{kg}$ \\
2-Butanone & ADS & & $\mathrm{U}$ & 9 & $\mu \mathrm{g} / \mathrm{kg}$ \\
2-Butanone & ADS & $\mathrm{R}$ & $\mathrm{U}$ & 9 & $\mu \mathrm{g} / \mathrm{kg}$ \\
2-Hexanone & ADS & & $\mathrm{U}$ & 9 & $\mu \mathrm{g} / \mathrm{kg}$ \\
2-Hexanone & ADS & $\mathrm{R}$ & $\mathrm{U}$ & 9 & $\mu \mathrm{g} / \mathrm{kg}$ \\
4-Methyl-2-Pentanone & ADS & & $\mathrm{U}$ & 9 & $\mu \mathrm{g} / \mathrm{kg}$ \\
4-Methyl-2-Pentanone & ADS & $\mathrm{R}$ & $\mathrm{U}$ & 9 & $\mu \mathrm{g} / \mathrm{kg}$
\end{tabular}


FHB013

Volatile Organic Analyses Continued:

\begin{tabular}{|c|c|c|c|c|c|}
\hline Analyte & Lab & Rep & Qualifier & Results & Units 1 \\
\hline Acetone & ADS & & $\mathrm{U}$ & 9 & $\mu \mathrm{g} / \mathrm{kg}$ \\
\hline Acetone & ADS & $\mathbf{R}$ & & 21 & $\mu \mathrm{g} / \mathrm{kg}$ \\
\hline Benzene & ADS & & $\mathrm{J}$ & 0 & $\mu \mathrm{g} / \mathrm{kg}$ \\
\hline Benzene & $\mathrm{ADS}$ & $\mathbf{R}$ & $\mathrm{J}$ & 0 & $\mu \mathrm{g} / \mathrm{kg}$ \\
\hline Bromodichloromethane & $\mathrm{ADS}$ & & $U$ & 9 & $\mu g / \mathrm{kg}$ \\
\hline Bromodichloromethane & ADS & $\mathbf{R}$ & $U$ & 9 & $\mu \mathrm{g} / \mathrm{kg}$ \\
\hline Bromoform & ADS & & $\mathrm{U}$ & 9 & $\mu \mathrm{g} / \mathrm{kg}$ \\
\hline Bromoform & ADS & $\mathrm{R}$ & $U$ & 9 & $\mu \mathrm{g} / \mathrm{kg}$ \\
\hline Bromomethane & ADS & & $U$ & 9 & $\mu \mathrm{g} / \mathrm{kg}$ \\
\hline Bromomethane & $\mathrm{ADS}$ & $\mathbf{R}$ & $\mathrm{U}$ & 9 & $\mu \mathrm{g} / \mathrm{kg}$ \\
\hline Carbon Disulfide & ADS & & $\mathrm{J}$ & 1 & $\mu g / \mathrm{kg}$ \\
\hline Carbon Disulfide & ADS & $\mathbf{R}$ & $\mathrm{J}$ & 1 & $\mu \mathrm{g} / \mathrm{kg}$ \\
\hline Carbon Tetrachloride & $\mathrm{ADS}$ & & $\mathrm{U}$ & 9 & $\mu \mathrm{g} / \mathrm{kg}$ \\
\hline Carbon Tetrachloride & $\mathrm{ADS}$ & $\mathbf{R}$ & $\mathrm{U}$ & 9 & $\mu \mathrm{g} / \mathrm{kg}$ \\
\hline Chlorobenzene & $\mathrm{ADS}$ & & $U$ & 9 & $\mu \mathrm{g} / \mathrm{kg}$ \\
\hline Chlorobenzene & ADS & $\mathrm{R}$ & $\mathrm{U}$ & 9 & $\mu \mathrm{g} / \mathrm{kg}$ \\
\hline Chloroethane & $\mathrm{ADS}$ & & $\mathrm{U}$ & 9 & $\mu \mathrm{g} / \mathrm{kg}$ \\
\hline Chloroethane & ADS & $\mathrm{R}$ & $\mathrm{J}$ & 1 & $\mu \mathrm{g} / \mathrm{kg}$ \\
\hline Chloroform & $\mathrm{ADS}$ & & J & 0 & $\mu \mathrm{g} / \mathrm{kg}$ \\
\hline Chloroform & ADS & $\mathrm{R}$ & $\mathrm{U}$ & 9 & $\mu \mathrm{g} / \mathrm{kg}$ \\
\hline Chloromethane & $\mathrm{ADS}$ & & $\mathrm{U}$ & 9 & $\mu \mathrm{g} / \mathrm{kg}$ \\
\hline Chloromethane & ADS & $\mathbf{R}$ & $U$ & 9 & $\mu g / \mathrm{kg}$ \\
\hline cis-1,2-Dichloroethene & $\mathrm{ADS}$ & & $\mathrm{U}$ & 9 & $\mu \mathrm{g} / \mathrm{kg}$ \\
\hline cis-1,2-Dichloroethene & ADS & $\mathrm{R}$ & $\mathrm{U}$ & 9 & $\mu \mathrm{g} / \mathrm{kg}$ \\
\hline cis-1,3-Dichloropropene & $\mathrm{ADS}$ & & $U$ & 9 & $\mu \mathrm{g} / \mathrm{kg}$ \\
\hline cis-1,3-Dichloropropene & ADS & $\mathbf{R}$ & $U$ & 9 & $\mu \mathrm{g} / \mathrm{kg}$ \\
\hline Dibromochloromethane & $\mathrm{ADS}$ & & $U$ & 9 & $\mu \mathrm{g} / \mathrm{kg}$ \\
\hline Dibromochloromethane & $\mathrm{ADS}$ & $\mathbf{R}$ & $\mathrm{U}$ & 9 & $\mu \mathrm{g} / \mathrm{kg}$ \\
\hline Ethylbenzene & ADS & & $\mathrm{U}$ & 9 & $\mu \mathrm{g} / \mathrm{kg}$ \\
\hline Ethylbenzene & ADS & $\mathrm{R}$ & $\mathbf{J}$ & 1 & $\mu \mathrm{g} / \mathrm{kg}$ \\
\hline Methylene Chloride & ADS & & $\mathrm{J}$ & 0 & $\mu \mathrm{g} / \mathrm{kg}$ \\
\hline Methylene Chloride & ADS & $\mathbf{R}$ & $\mathrm{U}$ & 9 & $\mu \mathrm{g} / \mathrm{kg}$ \\
\hline Styrene & ADS & & $\mathrm{J}$ & 0 & $\mu \mathrm{g} / \mathrm{kg}$ \\
\hline Styrene & ADS & $\mathbf{R}$ & $\mathrm{U}$ & 9 & $\mu \mathrm{g} / \mathrm{kg}$ \\
\hline Tetrachloroethene & ADS & & $J$ & 1 & $\mu \mathrm{g} / \mathrm{kg}$ \\
\hline Tetrachloroethene & ADS & $\mathbf{R}$ & $\mathrm{J}$ & 1 & $\mu \mathrm{g} / \mathrm{kg}$ \\
\hline Toluene & ADS & & $\mathrm{J}$ & 3 & $\mu \mathrm{g} / \mathrm{kg}$ \\
\hline Toluene & ADS & $\mathrm{R}$ & $\mathrm{J}$ & 6 & $\mu \mathrm{g} / \mathrm{kg}$ \\
\hline trans-1,2-Dichloroethene & ADS & & $\mathrm{U}$ & 9 & $\mu \mathrm{g} / \mathrm{kg}$ \\
\hline trans-1,2-Dichloroethene & $\mathrm{ADS}$ & $\mathrm{R}$ & $\mathbf{U}$ & 9 & $\mu \mathrm{g} / \mathrm{kg}$ \\
\hline trans-1,3-Dichloropropene & $\mathrm{ADS}$ & & $\mathrm{U}$ & 9 & $\mu \mathrm{g} / \mathrm{kg}$ \\
\hline trans-1,3-Dichloropropene & ADS & $\mathrm{R}$ & $\mathrm{U}$ & 9 & $\mu \mathrm{g} / \mathrm{kg}$ \\
\hline Trichloroethene & ADS & & $\mathrm{U}$ & 9 & $\mu \mathrm{g} / \mathrm{kg}$ \\
\hline Trichloroethene & ADS & $\mathrm{R}$ & $\mathrm{U}$ & 9 & $\mu \mathrm{g} / \mathrm{kg}$ \\
\hline Vinyl Acetate & ADS & & $U$ & 9 & $\mu \mathrm{g} / \mathrm{kg}$ \\
\hline Vinyl Acetate & ADS & $\mathrm{R}$ & $\mathrm{U}$ & 9 & $\mu \mathrm{g} / \mathrm{kg}$ \\
\hline
\end{tabular}




\section{FHB013}

Volatile Organic Analyses Continued:

\begin{tabular}{llcccc} 
Analyte & Lab & Rep & Qualifier & Results & Units 1 \\
\hline Vinyl Chloride & ADS & & $\mathrm{U}$ & 9 & $\mu \mathrm{g} / \mathrm{kg}$ \\
Vinyl Chloride & ADS & $\mathrm{R}$ & $\mathrm{U}$ & 9 & $\mu \mathrm{g} / \mathrm{kg}$ \\
Xylenes (Total) & ADS & & $\mathrm{U}$ & 9 & $\mu \mathrm{g} / \mathrm{kg}$ \\
Xylenes (Total) & ADS & $\mathrm{R}$ & $\mathrm{U}$ & 9 & $\mu \mathrm{g} / \mathrm{kg}$ \\
\hline
\end{tabular}

${ }^{1}$ Reported as wet weight.

Radiological Analyses:

\begin{tabular}{lllll} 
Analyte & Lab & Qualifier & Result & Units \\
\hline Gross Alpha & ADS & $\mathrm{J}$ & 2 & $\mathrm{pCi} / \mathrm{g}$ \\
Nonvolatile Beta & ADS & & 25 & $\mathrm{pCi} / \mathrm{g}^{2}$ \\
Tritium & ADS & & 580 & $\mathrm{pCi} / \mathrm{g}^{2}$ \\
\hline
\end{tabular}

Reported as dry weight after moisture content corrections.

2 Reported as wet weight. 


\section{FHBO14}

Sample Date: 03/07/94

Matrix: Soil

Moisture Content: $55.6 \%$

Metals Analyses:

\begin{tabular}{llccc} 
Analyte & Lab & Qualifier & Results & Units 1 \\
\hline Antimony & ADS & $\mathrm{U}$ & 56 & $\mu \mathrm{g} / \mathrm{g}$ \\
Arsenic & ADS & $\mathrm{U}$ & 56 & $\mu \mathrm{g} / \mathrm{g}$ \\
Barium & ADS & & 1.4 & $\mu \mathrm{g} / \mathrm{g}$ \\
Beryllium & ADS & & 0.84 & $\mu \mathrm{g} / \mathrm{g}$ \\
Cadmium & ADS & $\mathrm{U}$ & 3 & $\mu \mathrm{g} / \mathrm{g}$ \\
Chromium & ADS & $\mathrm{U}$ & 11 & $\mu \mathrm{g} / \mathrm{g}$ \\
Cobalt & ADS & $\mathrm{U}$ & 11 & $\mu \mathrm{g} / \mathrm{g}$ \\
Copper & ADS & $\mathrm{U}$ & 3 & $\mu \mathrm{g} / \mathrm{g}$ \\
Lead & ADS & $\mathrm{U}$ & 56 & $\mu \mathrm{g} / \mathrm{g}$ \\
Mercury & ADS & $\mathrm{U}$ & 3 & $\mu \mathrm{g} / \mathrm{g}$ \\
Nickel & ADS & $\mathrm{U}$ & 14 & $\mu \mathrm{g} / \mathrm{g}$ \\
Selenium & ADS & $\mathrm{U}$ & 84 & $\mu \mathrm{g} / \mathrm{g}$ \\
Silver & ADS & $\mathrm{U}$ & 6 & $\mu \mathrm{g} / \mathrm{g}$ \\
Thallium & ADS & $\mathrm{U}$ & 56 & $\mu \mathrm{g} / \mathrm{g}$ \\
Tin & ADS & $\mathrm{U}$ & 20 & $\mu \mathrm{g} / \mathrm{g}$ \\
Vanadium & ADS & $\mathrm{U}$ & 3 & $\mu \mathrm{g} / \mathrm{g}$ \\
Zinc & ADS & & 191 & $\mu \mathrm{g} / \mathrm{g}$ \\
\hline
\end{tabular}

$T_{\text {Reported as dry weight after moisture content corrections. }}$

Volatile Organic Analyses:

\begin{tabular}{|c|c|c|c|c|c|}
\hline Analyte & $\mathrm{Lab}$ & Rep & Qualifier & Results & Units 1 \\
\hline 1,1-Dichloroethene & $\mathrm{ADS}$ & & $\mathrm{U}$ & 9 & $\mu \mathrm{g} / \mathrm{kg}$ \\
\hline 1,1-Dichloroethane & $\mathrm{ADS}$ & & $\mathrm{U}$ & 9 & $\mu \mathrm{g} / \mathrm{kg}$ \\
\hline 1,1,1-Trichloroethane & $\mathrm{ADS}$ & & $\mathrm{U}$ & 9 & $\mu \mathrm{g} / \mathrm{kg}$ \\
\hline 1,2-Dichloroethane & ADS & & $U$ & 9 & $\mu \mathrm{g} / \mathrm{kg}$ \\
\hline 1,2-Dichloropropane & $\mathrm{ADS}$ & & $\mathrm{U}$ & 9 & $\mu \mathrm{g} / \mathrm{kg}$ \\
\hline 1,1,2-Trichloroethane & $\mathrm{ADS}$ & & $\mathrm{U}$ & 9 & $\mu \mathrm{g} / \mathrm{kg}$ \\
\hline 1,1,2,2-Tetrachloroethane & ADS & & $\mathrm{U}$ & 9 & $\mu \mathrm{g} / \mathrm{kg}$ \\
\hline 2-Butanone & ADS & & & 127 & $\mu \mathrm{g} / \mathrm{kg}$ \\
\hline 2-Hexanone & $\mathrm{ADS}$ & & $\mathrm{U}$ & 9 & $\mu \mathrm{g} / \mathrm{kg}$ \\
\hline 4-Methyl-2-Pentanone & ADS & & $U$ & 9 & $\mu \mathrm{g} / \mathrm{kg}$ \\
\hline Acetone & ADS & & & 137 & $\mu \mathrm{g} / \mathrm{kg}$ \\
\hline Benzene & ADS & & J & 0 & $\mu \mathrm{g} / \mathrm{kg}$ \\
\hline Bromodichloromethane & ADS & & $\mathrm{U}$ & 9 & $\mu \mathrm{g} / \mathrm{kg}$ \\
\hline Bromoform & ADS & & $U$ & 9 & $\mu \mathrm{g} / \mathrm{kg}$ \\
\hline Bromomethane & ADS & & $\mathrm{J}$ & 1 & $\mu \mathrm{g} / \mathrm{kg}$ \\
\hline Carbon Disulfide & ADS & & $\mathrm{J}$ & 2 & $\mu \mathrm{g} / \mathrm{kg}$ \\
\hline Carbon Tetrachloride & ADS & & $\mathrm{U}$ & 9 & $\mu \mathrm{g} / \mathrm{kg}$ \\
\hline Chlorobenzene & ADS & & $\mathrm{U}$ & 9 & $\mu \mathrm{g} / \mathrm{kg}$ \\
\hline Chloroethane & ADS & & $\mathrm{U}$ & 9 & $\mu \mathrm{g} / \mathrm{kg}$ \\
\hline Chloroform & ADS & & $\mathrm{U}$ & 9 & $\mu \mathrm{g} / \mathrm{kg}$ \\
\hline
\end{tabular}


FHB014

Volatile Organic Analyses Continued:

\begin{tabular}{|c|c|c|c|c|c|}
\hline Analyte & $\mathrm{Lab}$ & Rep & Qualifier & Results & Units 1 \\
\hline Chloromethane & ADS & & $\mathrm{J}$ & 8 & $\mu \mathrm{g} / \mathrm{kg}$ \\
\hline cis-1,2-Dichloroethene & ADS & & $\mathrm{J}$ & 8 & $\mu \mathrm{g} / \mathrm{kg}$ \\
\hline cis-1,3-Dichloropropene & ADS & & $\mathrm{U}$ & 9 & $\mu \mathrm{g} / \mathrm{kg}$ \\
\hline Dibromochloromethane & ADS & & $\mathrm{U}$ & 9 & $\mu \mathrm{g} / \mathrm{kg}$ \\
\hline Ethylbenzene & ADS & & $\mathrm{U}$ & 9 & $\mu \mathrm{g} / \mathrm{kg}$ \\
\hline Methylene Chloride & ADS & & $U$ & 9 & $\mu \mathrm{g} / \mathrm{kg}$ \\
\hline Styrene & ADS & & $\mathrm{U}$ & 9 & $\mu \mathrm{g} / \mathrm{kg}$ \\
\hline Tetrachloroethene & ADS & & $\mathrm{J}$ & 2 & $\mu \mathrm{g} / \mathrm{kg}$ \\
\hline Toluene & ADS & & $\mathrm{LJ}$ & 267 & $\mu \mathrm{g} / \mathrm{kg}$ \\
\hline trans-1,2-Dichloroethene & ADS & & $\mathrm{U}$ & 9 & $\mu \mathrm{g} / \mathrm{kg}$ \\
\hline trans-1,3-Dichloropropene & ADS & & $\mathrm{U}$ & 9 & $\mu \mathrm{g} / \mathrm{kg}$ \\
\hline Trichloroethene & ADS & & $\mathrm{J}$ & 2 & $\mu g / \mathrm{kg}$ \\
\hline Vinyl Acetate & $\mathrm{ADS}$ & & $\mathrm{U}$ & 9 & $\mu \mathrm{g} / \mathrm{kg}$ \\
\hline Vinyl Chloride & $\mathrm{ADS}$ & & $\mathrm{U}$ & 9 & $\mu \mathrm{g} / \mathrm{kg}$ \\
\hline Xylenes (Total) & ADS & & $\mathrm{U}$ & 9 & $\mu \mathrm{g} / \mathrm{kg}$ \\
\hline
\end{tabular}

TReported as wet weight.

Radiological Analyses:

\begin{tabular}{lllll} 
Analyte & Lab & Qualifier & Result & Units \\
\hline Gross Alpha & ADS & $\mathrm{J}$ & 3 & $\mathrm{pCi} / \mathrm{g}$ \\
Nonvolatile Beta & ADS & & 55 & $\mathrm{pCi} / \mathrm{g}$ \\
Tritium & ADS & & 730 & $\mathrm{pCi} / \mathrm{g}^{2}$ \\
\hline
\end{tabular}

IReported as dry weight after moisture content corrections.

2 Reported as wet weight. 


\section{FHB018}

Sample Date: 03/07/94

Matrix: Soil

Moisture Content: $41.1 \%$

Metals Analyses:

\begin{tabular}{llccc} 
Analyte & Lab & Qualifier & Results & Units 1 \\
\hline Antimony & ADS & & 77 & $\mu \mathrm{g} / \mathrm{g}$ \\
Arsenic & ADS & $\mathrm{U}$ & 37 & $\mu \mathrm{g} / \mathrm{g}$ \\
Barium & ADS & & 32 & $\mu \mathrm{g} / \mathrm{g}$ \\
Beryllium & ADS & & 0.37 & $\mu \mathrm{g} / \mathrm{g}$ \\
Cadmium & ADS & $\mathrm{U}$ & 2 & $\mu \mathrm{g} / \mathrm{g}$ \\
Chromium & ADS & $\mathrm{U}$ & 7 & $\mu \mathrm{g} / \mathrm{g}$ \\
Cobalt & ADS & $\mathrm{U}$ & 7 & $\mu \mathrm{g} / \mathrm{g}$ \\
Copper & ADS & $\mathrm{U}$ & 2 & $\mu \mathrm{g} / \mathrm{g}$ \\
Lead & ADS & $\mathrm{U}$ & 37 & $\mu \mathrm{g} / \mathrm{g}$ \\
Mercury & ADS & $\mathrm{U}$ & 2 & $\mu \mathrm{g} / \mathrm{g}$ \\
Nickel & ADS & $\mathrm{U}$ & 9 & $\mu \mathrm{g} / \mathrm{g}$ \\
Selenium & ADS & $\mathrm{U}$ & 55 & $\mu \mathrm{g} / \mathrm{g}$ \\
Silver & ADS & $\mathrm{U}$ & 4 & $\mu \mathrm{g} / \mathrm{g}$ \\
Thallium & ADS & $\mathrm{U}$ & 37 & $\mu \mathrm{g} / \mathrm{g}$ \\
Tin & ADS & $\mathrm{U}$ & 13 & $\mu \mathrm{g} / \mathrm{g}$ \\
Vanadium & ADS & $\mathrm{U}$ & 2 & $\mu \mathrm{g} / \mathrm{g}$ \\
Zinc & ADS & & 160 & $\mu \mathrm{g} / \mathrm{g}$ \\
\hline
\end{tabular}

TReported as dry weight after moisture content corrections.

Volatile Organic Analyses:

\begin{tabular}{|c|c|c|c|c|c|}
\hline Analyte & $\mathrm{Lab}$ & $\operatorname{Rep}$ & Qualifier & Results & Units 1 \\
\hline 1,1-Dichloroethene & ADS & & $\mathrm{U}$ & 9 & $\mu \mathrm{g} / \mathrm{g}$ \\
\hline 1,1-Dichloroethane & $\mathrm{ADS}$ & & $\mathrm{U}$ & 9 & $\mu \mathrm{g} / \mathrm{g}$ \\
\hline 1,1,1-Trichloroethane & ADS & & $U$ & 9 & $\mu \mathrm{g} / \mathrm{g}$ \\
\hline 1,2-Dichloroethane & $\mathrm{ADS}$ & & $\mathrm{U}$ & 9 & $\mu \mathrm{g} / \mathrm{g}$ \\
\hline 1,2-Dichloropropane & $\mathrm{ADS}$ & & $\mathrm{U}$ & 9 & $\mu \mathrm{g} / \mathrm{g}$ \\
\hline 1,1,2-Trichloroethane & $\mathrm{ADS}$ & & $U$ & 9 & $\mu \mathrm{g} / \mathrm{g}$ \\
\hline 1,1,2,2-Tetrachloroethane & ADS & & $\mathrm{U}$ & 9 & $\mu g / g$ \\
\hline 2-Butanone & ADS & & & 20 & $\mu \mathrm{g} / \mathrm{g}$ \\
\hline 2-Hexanone & $\mathrm{ADS}$ & & $\mathrm{U}$ & 9 & $\mu \mathrm{g} / \mathrm{g}$ \\
\hline 4-Methyl-2-Pentanone & $\mathrm{ADS}$ & & $\mathrm{U}$ & 9 & $\mu \mathrm{g} / \mathrm{g}$ \\
\hline Acetone & $\mathrm{ADS}$ & & & 70 & $\mu \mathrm{g} / \mathrm{g}$ \\
\hline Benzene & $\mathrm{ADS}$ & & $\mathrm{J}$ & 1 & $\mu \mathrm{g} / \mathrm{g}$ \\
\hline Bromodichloromethane & ADS & & U & 9 & $\mu \mathrm{g} / \mathrm{g}$ \\
\hline Bromoform & ADS & & $\mathrm{U}$ & 9 & $\mu \mathrm{g} / \mathrm{g}$ \\
\hline Bromomethane & $\mathrm{ADS}$ & & $\mathrm{J}$ & 2 & $\mu \mathrm{g} / \mathrm{g}$ \\
\hline Carbon Disulfide & $\mathrm{ADS}$ & & $\mathrm{J}$ & 2 & $\mu \mathrm{g} / \mathrm{g}$ \\
\hline Carbon Tetrachloride & ADS & & $\mathrm{U}$ & 9 & $\mu \mathrm{g} / \mathrm{g}$ \\
\hline Chlorobenzene & ADS & & $U$ & 9 & $\mu \mathrm{g} / \mathrm{g}$ \\
\hline Chloroethane & ADS & & $\mathrm{U}$ & 9 & $\mu g / g$ \\
\hline Chloroform & ADS & & $\mathrm{U}$ & 9 & $\mu \mathrm{g} / \mathrm{g}$ \\
\hline
\end{tabular}




\section{FHB018}

Volatile Organic Analyses Continued:

\begin{tabular}{|c|c|c|c|c|c|}
\hline Analyte & Lab & Rep & Qualifier & Results & Units ${ }^{1}$ \\
\hline Chloromethane & ADS & & & 16 & $\mu \mathrm{g} / \mathrm{g}$ \\
\hline cis-1,2-Dichloroethene & ADS & & $\mathrm{U}$ & 9 & $\mu g / g$ \\
\hline cis-1,3-Dichloropropene & ADS & & $\mathrm{U}$ & 9 & $\mu \mathrm{g} / \mathrm{g}$ \\
\hline Dibromochloromethane & ADS & & $U$ & 9 & $\mu \mathrm{g} / \mathrm{g}$ \\
\hline Ethylbenzene & ADS & & $\mathrm{U}$ & 9 & $\mu \mathrm{g} / \mathrm{g}$ \\
\hline Methylene Chloride & ADS & & $\mathrm{U}$ & 9 & $\mu \mathrm{g} / \mathrm{g}$ \\
\hline Styrene & ADS & & $U$ & 9 & $\mu g / g$ \\
\hline Tetrachloroethene & ADS & & $J$ & 1 & $\mu g / g$ \\
\hline Toluene & ADS & & & 37 & $\mu \mathrm{g} / \mathrm{g}$ \\
\hline trans-1,2-Dichloroethene & $\mathrm{ADS}$ & & J & 0 & $\mu g / g$ \\
\hline trans-1,3-Dichloropropene & ADS & & $\mathrm{U}$ & 9 & $\mu \mathrm{g} / \mathrm{g}$ \\
\hline Trichloroethene & ADS & & $\mathrm{U}$ & 9 & $\mu g / g$ \\
\hline Vinyl Acetate & ADS & & $\mathbf{U}$ & 9 & $\mu g / g$ \\
\hline Vinyl Chloride & ADS & & $\mathrm{U}$ & 9 & $\mu \mathrm{g} / \mathrm{g}$ \\
\hline Xylenes (Total) & $\mathrm{ADS}$ & & $\mathrm{U}$ & 9 & $\mu \mathrm{g} / \mathrm{g}$ \\
\hline
\end{tabular}

${ }_{\text {Reported as wet weight. }}$

Radiological Analyses:

\begin{tabular}{lllll} 
Analyte & Lab & Qualifier & Result & Units \\
\hline Gross Alpha & ADS & 8 & $\mathrm{pCi} / \mathrm{g}$ \\
Nonvolatile Beta & ADS & 40 & $\mathrm{pCi} / \mathrm{g}$ \\
Tritium & ADS & 19900 & $\mathrm{pCi} / \mathrm{g}$ \\
\hline
\end{tabular}

Teported as dry weight after moisture content corrections.

2 Reported as wet weight. 